\title{
Rayleigh type behavior of the Young's modulus of unpoled ferroelectric ceramics and its dependence on temperature
}

\author{
M. Algueró, ${ }^{\text {a) }}$ B. Jiménez, and L. Pardo \\ Instituto de Ciencia de Materiales de Madrid, CSIC, Cantoblanco, 28049 Madrid, Spain
}

(Received 23 April 2003; accepted 29 July 2003)

\begin{abstract}
The dependence on stress of the low frequency Young's modulus and mechanical losses of unpoled ferroelectric ceramics has been studied as a function of temperature. The Young's modulus of unpoled $\mathrm{Pb}(\mathrm{Zr}, \mathrm{Ti}) \mathrm{O}_{3}$ (PZT) showed a Rayleigh type dependence, analogous to the one already described for the longitudinal piezoelectric coefficient. This has been associated to ferroelectric/ ferroelastic domain wall movements across, and their pinning/depinning on, randomly distributed defects. The Rayleigh coefficient was found to increase with temperature. The activation energy of the Rayleigh process was obtained, which must be related to the pinning energy. The Young's modulus of $\mathrm{Mn}$ doped $0.65 \mathrm{~Pb}\left(\mathrm{Mg}_{1 / 3} \mathrm{Nb}_{2 / 3}\right) \mathrm{O}_{3}-0.35 \mathrm{PbTiO}_{3}$ showed no stress dependence. (C) 2003 American Institute of Physics. [DOI: 10.1063/1.1614414]
\end{abstract}

Ferroelectric perovskite lead zirconate titanate (PZT) based piezoelectric ceramics are widely used in electromechanical transduction applications. ${ }^{1}$ Their piezoelectric coefficients are known to comprise a ferroelectric/ferroelastic domain wall contribution, which can account for more than $60 \%$ of the response at room temperature. ${ }^{2}$ Polarization reorientation above the coercive field occurs through domain wall movements. ${ }^{3}$ The intermediate regime between linearity and polarization reorientation has not received much attention until recently, when fields that were a significant fraction of the coercive field started to be used. ${ }^{4}$ Such fields cause the appearance of nonlinearities and hysteresis. ${ }^{5,6}$ It has been shown that there is a field range after linearity breakdown, in which the longitudinal piezoelectric coefficient, $d_{33}$, increases with field according to: ${ }^{7}$

$$
d_{33}(F)=d_{33}(0)+\alpha F,
$$

where $d_{33}(0)$ is the constant piezoelectric coefficient, $\alpha$ the nonlinear coefficient, and $F$ indicates field, which can be either the mechanical stress, $\sigma$, or the electric field, $E$. It has also been shown that both $d_{33}(0)$ and $\alpha$ present a logarithmic frequency dependence. ${ }^{8,9}$ This behavior is analogous to that of the magnetic susceptibility for ferromagnetic materials (Rayleigh law), which has been associated with the movement of ferromagnetic domain walls across, and their pinning/depinning on, a field of randomly distributed defects. The same mechanism, in this case involving ferroelectric/ ferroelastic domain walls, has been proposed to cause the linear dependence of the piezoelectric coefficient on field. ${ }^{7-9}$ The formalism has been used to study the effect of grain size and crystal structure on domain wall mobility for several ferroelectrics. ${ }^{10,11}$ However, the use of the piezoelectric coefficient requires poled samples, and it is difficult to achieve the same electrical polarization for samples with, for instance, different microstructure. Also, studies with temperature are limited by thermal depolarization. Addressing these effects requires studying unpoled samples. This can be done

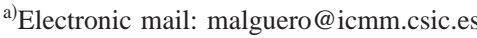

with the dielectric permittivity. Rayleigh type behavior of the permittivity has been reported for ceramics ${ }^{12}$ and films, ${ }^{13}$ but both $180^{\circ}$ ferroelectric and non- $180^{\circ}$ ferroelectric/ ferroelastic domain walls contribute to the coefficient. An alternative are elastic constants, to which only ferroelectric/ ferroelastic walls contribute.

Dynamical mechanical analysis (DMA) in three points bending (TPB) configuration has been used here to obtain the low frequency Young's modulus and mechanical losses of unpoled ferroelectric ceramics as a function of stress and temperature. ${ }^{14}$ This parameter has been shown to reflect $90^{\circ}$ domain wall movements in tetragonal PZT. ${ }^{15}$ A stress sine wave of variable amplitude (6-14 MPa) and $9 \mathrm{~Hz}$ frequency, superimposed on a static stress of $15 \mathrm{MPa}$ was applied to bending ceramic bars of $12 \times 1.5 \times 0.5 \mathrm{~mm}^{3}$ dimensions. In DMA/TPB, the Young's modulus, $Y$, is obtained from the amplitude of the bar flexure, and the mechanical losses, $\tan \delta$, from the lag time between the stress and flexural waves. Measurements were accomplished from room temperature to $450{ }^{\circ} \mathrm{C}$ at $3{ }^{\circ} \mathrm{C} \mathrm{min}^{-1}$ on heating and cooling. Two successive heating/cooling cycles were done for each measurement, the second being recorded.

Two high piezoelectric coefficient materials were characterized: a "soft" with donor cation substitution to increase the ferroelectric/ferroelastic domain wall mobility- ${ }^{1}$ PZT based commercial piezoceramic (PZ27 from Ferroperm Piezoceramics $\mathrm{A} / \mathrm{S}, d_{33}=425 \mathrm{pC} \mathrm{N}^{-1}, \quad \varepsilon_{33}^{\sigma}=1800 \varepsilon_{o}$, and $\tan \delta_{\varepsilon}=0.017, \quad T_{c}=350^{\circ} \mathrm{C}$ ), and $\mathrm{Mn}$ doped $0.65 \mathrm{~Pb}\left(\mathrm{Mg}_{1 / 3} \mathrm{Nb}_{2 / 3}\right) \mathrm{O}_{3}-0.35 \mathrm{PbTiO}_{3}$ (from Thales R\&T, $d_{33}$ $=486 \mathrm{pC} \mathrm{N}^{-1}, \quad \varepsilon_{33}^{\sigma}=4206 \varepsilon_{o} \quad$ and $\tan \delta_{\varepsilon}=0.014, \quad T_{c}$ $\left.=169^{\circ} \mathrm{C}\right)$. This latter relaxor based ferroelectric (PMN-PT) has gained much attention after the report of ultrahigh piezoelectricity and electric field induced strain in single crystals ${ }^{16}$ and textured ceramics, ${ }^{17}$ and the deformation mechanisms are under intense investigation. Both compositions are close to the morphotropic phase boundary between the rhombohedral and tetragonal phases of their respective solid solutions. ${ }^{18,19}$ Therefore, they present tetragonal $90^{\circ}$ and rhombohedral $71^{\circ}$ and $109^{\circ}$ ferroelectric/ferroelastic domain walls. 

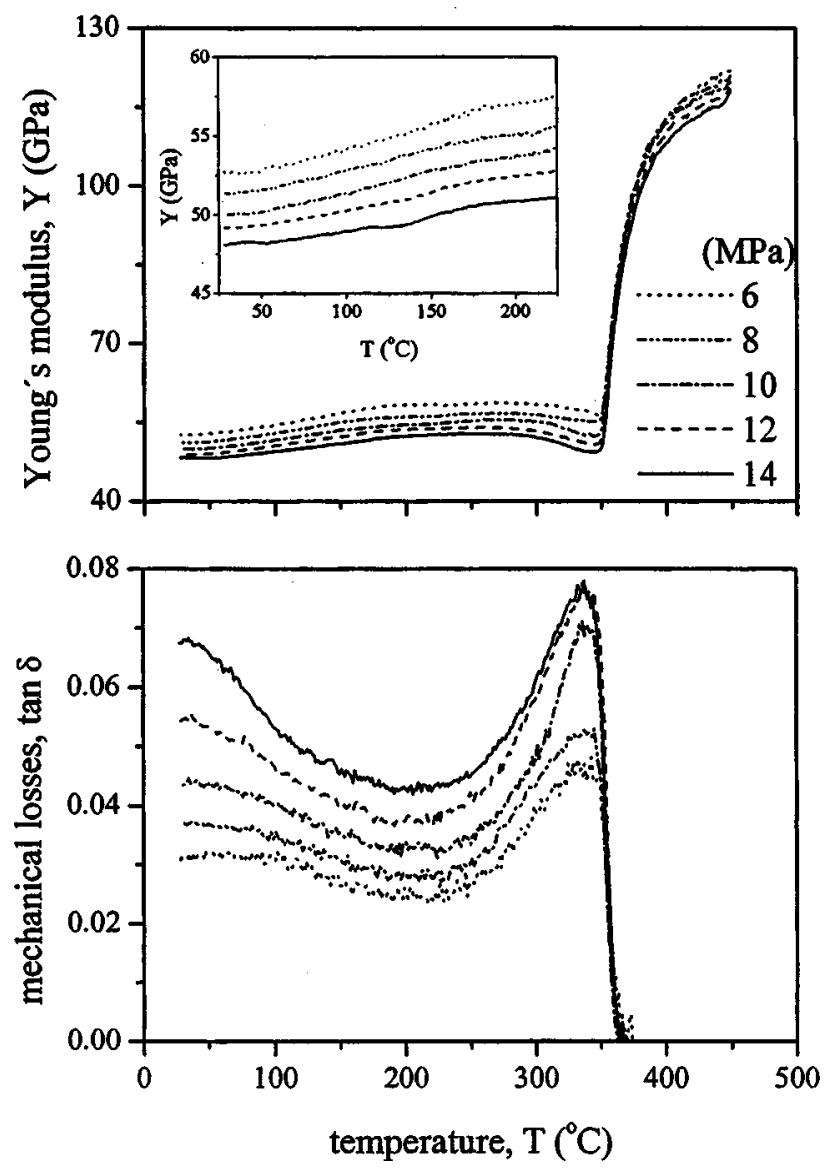

FIG. 1. Low frequency Young's modulus and mechanical losses of PZ27 unpoled ceramics as a function of temperature for several stresses.

Figure 1 shows the Young's modulus and mechanical losses of unpoled PZT as a function of temperature for increasing stresses. The sharp stiffening at $353^{\circ} \mathrm{C}$ is related to the ferroelectric-paraelectric phase transition. This occurs along with a strong reduction of the losses associated with the disappearance of the ferroelectric/ferroelastic domain structure. ${ }^{14}$ Mechanical losses were negligible in the paraelectric phase, which indicates that all losses in the ferroelectric phase were linked to domain wall movements. The phase transition was preceded by an increase of the domain wall activity that started at $\sim 250{ }^{\circ} \mathrm{C}$, as shown by the broad loss peak just below the transition temperature. Another loss peak was observed at $46^{\circ} \mathrm{C}$. Additional measurements at 3 and $21 \mathrm{~Hz}$ showed that the peak did not significantly shift with frequency. This peak might originate from a rhombohedral to tetragonal phase transition under the measuring stresses. The Young's modulus of the ferroelectric phase showed a clear dependence on stress, decreasing as the stress increased. The inset in the figure shows a magnification of the temperature range in which the Rayleigh type analysis was carried out. Temperatures up to $200^{\circ} \mathrm{C}$ were analyzed, well before rearrangement of the domain assemblage occurred. Figure 2 shows the dependence of the Young's modulus on stress for five temperatures (out of the ten analyzed, for clarity). Linear fits to the Rayleigh type expression:

$$
Y(\sigma)=Y(0)+\alpha \sigma,
$$

are also shown. Correlation factors higher than 0.994 were obtained for all temperatures, which indicates that Eq. (2) Downloaded 19 Feb 2010 to 161.111 .180 .191 . Redistribution subje

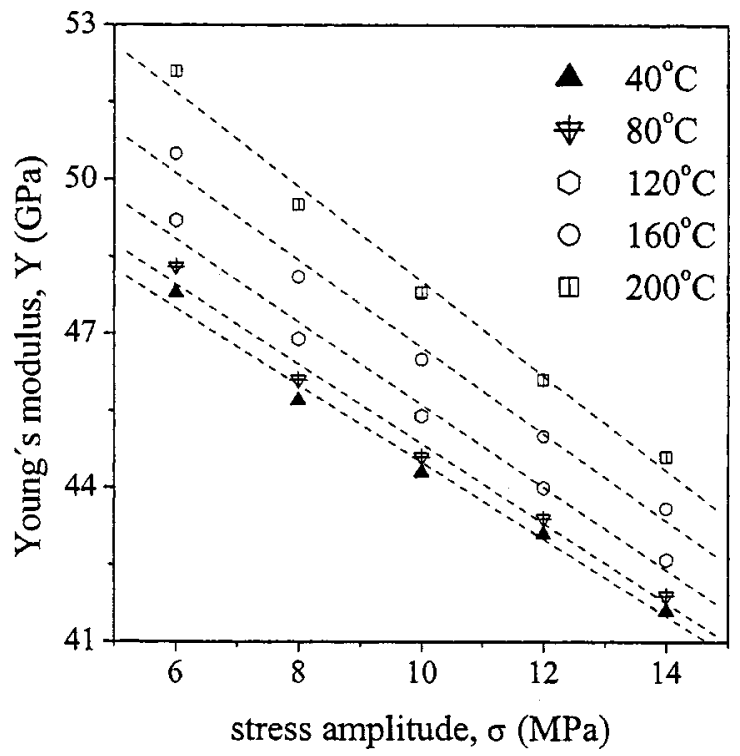

FIG. 2. Rayleigh type analysis of the stress dependence of the low frequency Young's modulus of unpoled PZ27 for several temperatures.

describes the stress dependence well. An increase of the Young's modulus with frequency was also observed. This strongly indicates that the Young's modulus of the unpoled ceramic is reflecting the movement of the ferroelectric/ ferroelastic domain walls and their interaction with the existing defects. The constant Young's modulus, $Y(0)$, showed a significant scattering between samples [33-56 GPa at room temperature (RT)], which is most probably a consequence of microcracking introduced during the machining of the bending bars. On the other hand, this magnitude showed a very consistent trend with temperature for all the samples, increasing $\sim 10 \%$ from RT to $200{ }^{\circ} \mathrm{C}$. Rayleigh coefficients, $\alpha$, between -550 and -750 were obtained at RT. The temperature dependence of the coefficient is shown in Fig. 3 for three samples. An Arrhenius analysis was accomplished for obtaining the activation energy of the Rayleigh process. Two regimes were found. A low temperature regime with an acti-

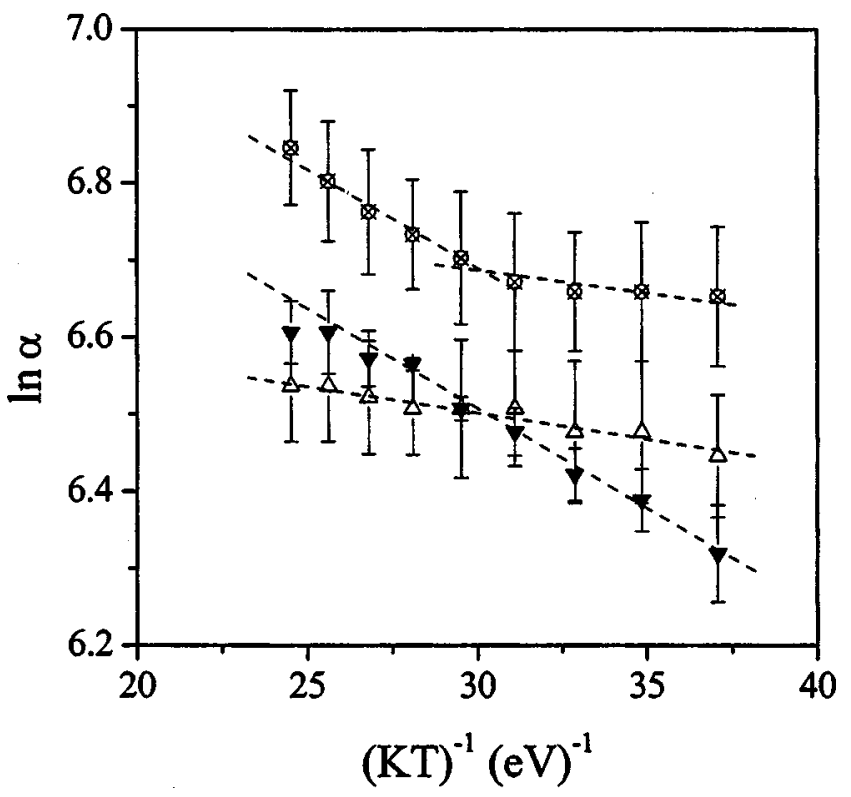

FIG. 3. Arrhenius type analysis of the temperature dependence of the Rayleigh coefficient for three PZ27 unpoled ceramic samples.

to AIP license or copyright; see http://apl.aip.org/apl/copyright.jsp 

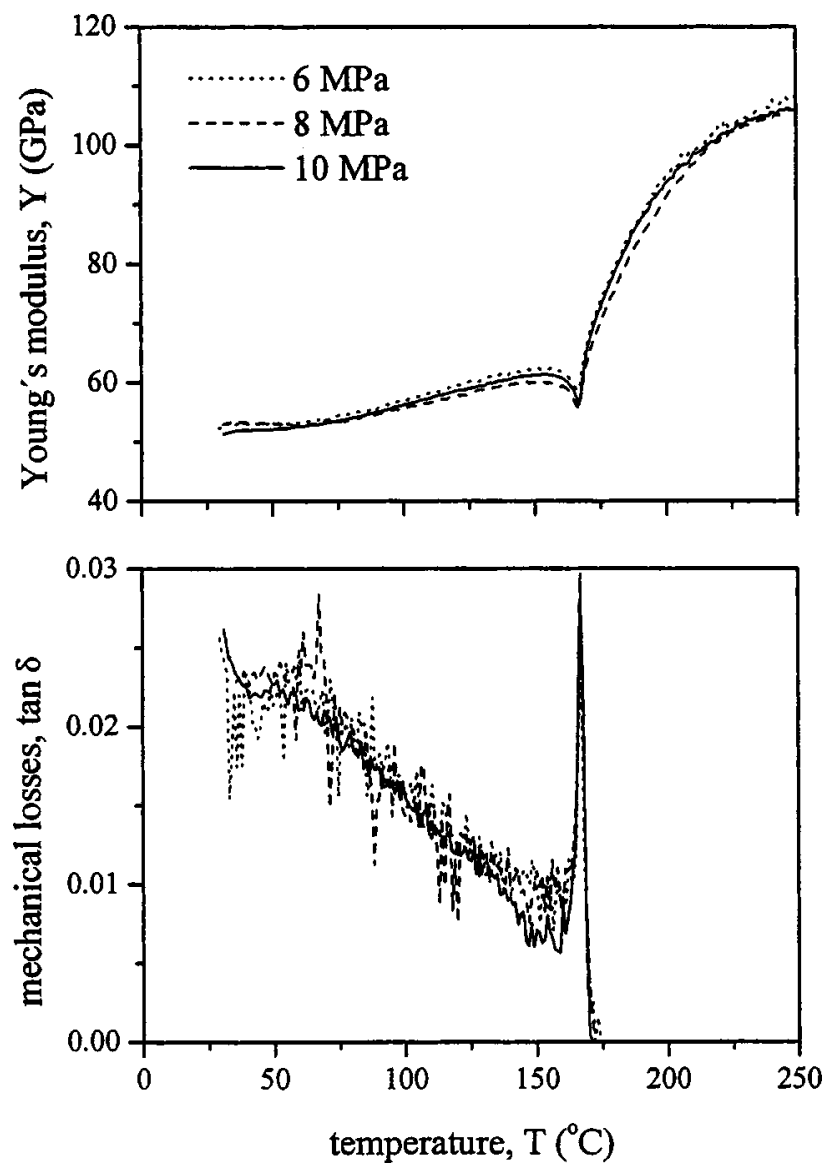

FIG. 4. Low frequency Young's modulus and mechanical losses of 0.65PMN-0.35PT:Mn unpoled ceramics as a function of temperature for several stresses.

vation energy of $8 \pm 2 \mathrm{meV}$, and a high temperature one with an activation energy of $25 \pm 3 \mathrm{meV}$. The temperature at which the high temperature regime appeared showed significant scattering as illustrated in the figure. One sample showed no high temperature regime, another one showed only the high temperature regime, and the third sample, a change from low to high at $120^{\circ} \mathrm{C}$. The Rayleigh behavior has been said to be associated with the movement of the domain walls across a field of randomly distributed defects, with which they interact, in a continuous pinning/depinning process. In other words, defects introduce irregular perturbations of the potential energy of the moving domain wall. ${ }^{9}$ Therefore, the obtained activation energies are the average height of the local potential irregularities, and are related to the pinning energy.

The nature of the defects and of their interaction with the walls has not been fully discussed. Donor doped compositions are usually formulated with cation vacancies, which could be the pinning defects. The Rayleigh process is a particular case of the more general Preisach formalism that predicts a quadratic dependence of the coefficients (piezoelectric, dielectric, and elastic) on field. ${ }^{20}$ This formalism assumes the existence of a large ensemble of microscopic bistable units characterized by distributed internal and coercive fields. Vacancies may produce such a distribution of fields. The existence of two activation energies could be related to the coexistence of two phases with different crystallographic distortions of the perovskite and the presence of three different types of domains. Rhombohedral ferroelectric/ferroelastic domain walls should be easier to move than tetragonal walls as the strain energy involved is smaller.

Figure 4 shows the Young's modulus and mechanical losses of unpoled PMN-PT as a function of temperature for increasing stresses. Again, the stiffening at $167^{\circ} \mathrm{C}$ is associated with the ferroelectric-paraelectric phase transition, and like PZT, is accompanied by the disappearance of all losses. Unlike PZT, neither the Young's modulus nor the losses in the ferroelectric phase showed a stress dependence. The absence of Rayleigh type behavior for Aurivillius $\mathrm{SrBi}_{4} \mathrm{Ti}_{4} \mathrm{O}_{15}$ (SBT) has been related to the walls being immobile. ${ }^{7}$ This does not seem to be case for PMN-PT, because nonnegligible losses, though smaller than for PZT, exist indicating some type of domain wall motion. Viscous-like movement of domain walls is likely occurring. ${ }^{21}$

In summary, the Young's modulus of unpoled PZT ceramics shows Rayleigh type behavior, and is therefore a suitable coefficient for studying the effect on the ferroelectric/ ferroelastic domain wall mobility of different parameters that are difficult to control in poled samples. This has been done as a function of temperature, and the activation energy of the Rayleigh process, which must be the average height of the irregularities of the potential energy under which the walls move, has been obtained. PMN-PT has also been characterized, and showed no Rayleigh behavior.

This work has been funded by the European Commision (PIRAMID Growth Project No. G5RD-CT-2001-00456) and the Spanish McyT (Acción Especial MAT2001-4819-E and Project No. MAT2002-00463).

${ }^{1}$ K. Uchino, Acta Mater. 46, 3745 (1998).

${ }^{2}$ Q. M. Zhang, H. Wang, N. Kim, and L. E. Cross, J. Appl. Phys. 75, 454 (1994).

${ }^{3}$ S. C. Hwang, C. S. Lynch, and R. M. McMeeking, Acta Metall. Mater. 43, 2073 (1995)

${ }^{4}$ K. Uchino, Mater. Res. Bull. 18, 42 (1993).

${ }^{5}$ Q. M. Zhang, W. Y. Pan, S. J. Jang, and L. Cross, J. Appl. Phys. 64, 6445 (1988).

${ }^{6}$ S. Li, W. Cao, and L. E. Cross, J. Appl. Phys. 69, 7219 (1991).

${ }^{7}$ D. Damjanovic and M. Demartin, J. Phys. D 29, 2057 (1996).

${ }^{8}$ D. Damjanovic, Phys. Rev. B 55, R649 (1997).

${ }^{9}$ D. Damjanovic, J. Appl. Phys. 82, 1788 (1997).

${ }^{10}$ M. Demartin and D. Damjanovic, Appl. Phys. Lett. 68, 3046 (1996).

${ }^{11}$ D. Damjanovic and M. Demartin, J. Phys.: Condens. Matter 9, 4943 (1997).

${ }^{12}$ D. A. Hall, M. M. Ben-Omran, and P. J. Stevenson, J. Phys.: Condens. Matter 10, 461 (1998).

${ }^{13}$ D. V. Taylor and D. Damjanovic, Appl. Phys. Lett. 73, 2045 (1998).

${ }^{14}$ B. Jiménez and J. M. Vicente, J. Phys. D 31, 130 (1998).

${ }^{15}$ B. Jiménez and J. M. Vicente, J. Phys. D 33, 1525 (2000).

${ }^{16}$ S. E. Park and T. R. Shrout, J. Appl. Phys. 82, 1804 (1997).

${ }^{17}$ E. M. Sabolsky, A. R. James, S. Kwon, S. Trolier-McKinstry, and G. L. Messing, Appl. Phys. Lett. 78, 2551 (2001).

${ }^{18}$ W. Cao and L. E. Cross, Phys. Rev. B 47, 4825 (1993).

${ }^{19}$ O. Noblanc, P. Gaucher, and G. Calvarin, J. Appl. Phys. 79, 4291 (1996).

${ }^{20}$ G. Roberts, D. Damjanovic, and N. Setter, J. Appl. Phys. 90, 2459 (2001).

${ }^{21}$ G. Roberts, D. Damjanovic, and N. Setter, J. Appl. Phys. 90, 4668 (2001). 